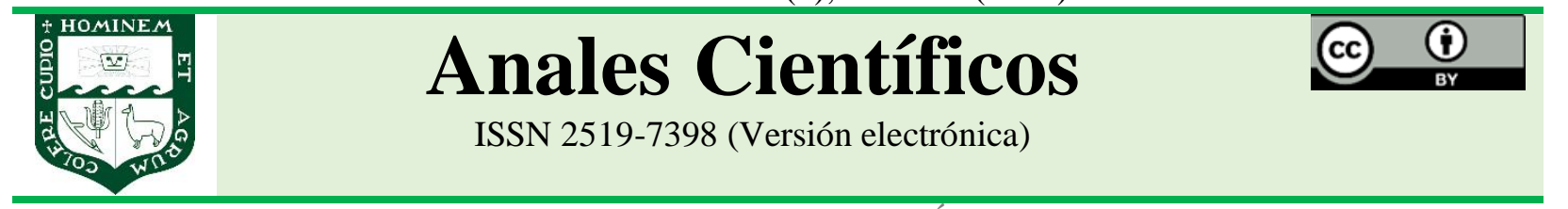

ARTÍCULO ORIGINAL - RESEARCH ARTICLE

http://dx.doi.org/10.21704/ac.v82i1.1752

\title{
CARACTERIZACIÓN DE UN PAQUETE TECNOLÓGICO PARA EL PROCESAMIENTO DE CAFÉ VERDE PERUANO CON CARACTERÍSTICAS DE CAFÉS ESPECIALES PARA EXPORTACIÓN
}

\section{Characterization of a technology package for the Peruvian green coffee processing with specialty coffee qualities to export}

\author{
Ibeth D. Pajuelo Tucto ${ }^{1 *} \mathbb{1}$; Luis E. Huerta Camones ${ }^{1}[0$ \\ ${ }^{1}$ Facultad de Economía y Planificación, Universidad Nacional Agraria La Molina, La Molina, 15024, Lima, Perú. \\ *E-mail:20130258@lamolina.edu.pe
}

Recibido: 20/07/2020; Aceptado: 21/07/2021; Publicado: 31/08/2021

\begin{abstract}
The objective of this study was to define a technology package, in order to provide an inventory of the technologies used during the processing of the Peruvian green coffee with specialty coffee qualities to export, supported by a technological management perspective. The methodology relied on a technology package construction, by identifying, describing and quantifying four of its components, based on a systematic literature review, and five professional's endorsements; each of them knowledgeable of the technologies used in agricultural products processing. Here, semi-structured interviews (Massachusetts Institute of Technology protocol) were used. As a result, a technology package was obtained with 79 generic technologies, 76 of these are involved during the processing activities, which develop physical, sensorial and cultural specialty coffee qualities, valuable for foreign markets. The package defined has limitations related to technical specifications. However, the study reveals its possible applications to: diagnose the management of current technologies, take decisions according to the specific case needs, and boost a technological forthcoming approach to match technologies with market requirements.
\end{abstract}

Keywords: product technology; equipment technology; process technology; operation technology; technology management

\section{RESUMEN}

El objetivo de este estudio fue definir un paquete tecnológico que permita inventariar las tecnologías utilizadas en el procesamiento de café verde peruano con características de café especial para exportación bajo el enfoque de la gestión tecnológica. El método utilizado se basó en la conformación de un paquete tecnológico mediante la identificación, descripción y cuantificación de sus cuatro componentes, a partir de una revisión sistemática de la literatura y sustentación de cinco profesionales, conocedores de las tecnologías de procesamiento para productos agrícolas, por medio de entrevistas semiestructurada (protocolo del Instituto Tecnológico de Massachusetts). Como resultado se obtuvo un paquete tecnológico con 79 tecnologías genéricas, de las cuales 76 intervienen durante el procesamiento para generar características físicas, sensoriales y culturales de cafés especiales, valoradas en el mercado internacional. El paquete definido presenta limitaciones en sus especificaciones técnicas. Sin embargo, el estudio revela sus posibles usos: diagnosticar la gestión de la tecnología existente, tomar de decisiones de acuerdo 
Pajuelo, I. \& Huerta, L. (2021). Anales Científicos. 82(1), 171-179. DOI. 10.21704/ac.v82i1.1752

con las necesidades específicas del caso y generar una prospectiva tecnológica que permita calzar las tecnologías con los requerimientos de la demanda.

Palabras clave: tecnología de producto; tecnología de equipo; tecnología de proceso; tecnología de operación; gestión tecnológica

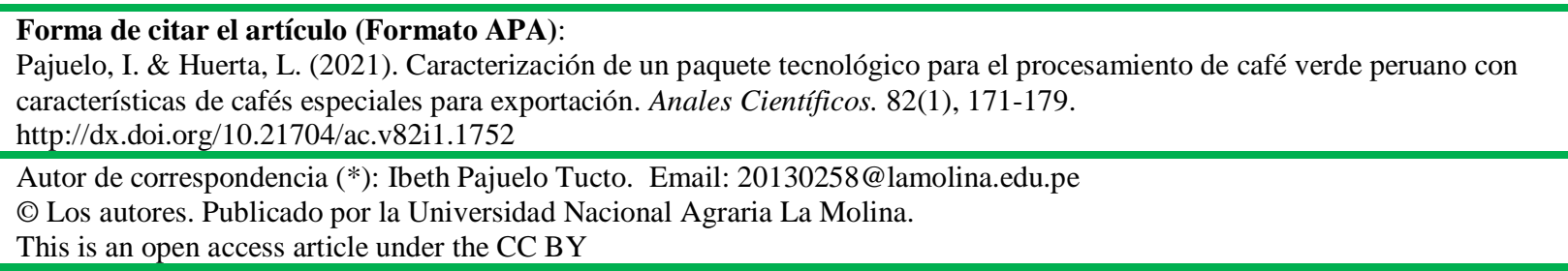

\section{INTRODUCCIÓN}

Para la gestión tecnológica empresarial, el paquete tecnológico es el conjunto de todos los conocimientos necesarios que se estructura para generar productos o servicios, así como procesos superiores para atender la demanda (Solleiro y Castañón, 2016). Conocer sus componentes, permite a los usuarios analizar opciones y tomar una decisión preliminar sobre cuál tecnología adoptar para producir bienes con características particulares preespecificadas (Delgado, 2009; Zarazúa-Solleiro et al., 2009; Napaico, 2015). Para la exportación de café verde peruano (grano obtenido al final del procesamiento del fruto), se seleccionan las características que les permitan negociar un mejor precio en el comercio internacional (Palacios, 2014).

Actualmente, se priorizan las características del café especial (físicas, sensoriales y culturales) por su valor creciente en el mercado, donde los tostadores incrementan su influencia principalmente enfocados en la calidad (Grabs y Ponte, 2019). Para asegurar la calidad del producto final y expresar lo mejor del grano para el comprador, es importante que se maneje durante las operaciones de su procesamiento con el paquete tecnológico adecuado (Rhinehart, 2009; Puerta, 2011; Dávila, 2018).

De acuerdo con Bitzer-Glasbergen et al. (2013) en el Perú, los programas de difusión tecnológica en el sector cafetalero para mejorar las características del café, comprende actividades orientadas al mejoramiento de las condiciones de poscosecha, implementación de un sistema de producción orgánica y participación en concursos anuales de calidad del café. En la zona de siembra y cosecha de café, el Instituto Nacional de Innovación Agraria (INIA) se encarga de transferir un paquete tecnológico integrado por tecnologías agrarias: variedad, método de control, procedimiento, fórmula de fertilización, oportunidad de aplicación de un agroquímico, un método de poda, manejo pre o post cosecha, etc. (INIA, 2016).

Por otro lado, en los Centros de Innovación Productiva y Transferencia Tecnológica (CITE) relacionados al café como los socios tecnológicos de las empresas para promover innovaciones que permitan aprovechar las oportunidades de los acuerdos comerciales, proveen servicios tecnológicos que incluyen equipamiento para el procesamiento de la materia prima, personal especializado como ingenieros en industrias alimentarias, laboratorios para análisis físico y microbiológico, y capacitaciones para el desarrollo de nuevos productos, pero aún no se conoce un paquete tecnológico en la agroindustria que permita generar valor agregado (OECD, 2011; PNUD, 2017). Asimismo, el Ministerio de Agricultura y Riego (MINAGRI) propuso el Plan Nacional de Acción del Café Peruano 2018 - 2030. con la visión: “Al 2030, el Perú es un país productor, exportador y consumidor de café sostenible de calidad, adaptado al cambio climático, reconocido a nivel mundial por su innovación, competitividad y una sólida institucionalidad que beneficia directamente a las familias cafetaleras y al conjunto de actores" (MINAGRI, 2018), pero ha sido considerado como "irrealista" en términos técnicos y financieros por la JNC (Junta Nacional del Café), integrada por 56 organizaciones cafetaleras (JNC, 2019). 
Por otra parte, los gerentes de las organizaciones agroexportadoras de café, afirman conocer la demanda, pero contar con poca información técnica para mejorar su oferta (Aire, 2019).

Por consiguiente, en el sector cafetalero peruano, aún se necesitan generar conocimientos oportunos y pertinentes que sean transferidos, validados y adoptados para mantener su competitividad frente a países como Brasil y Colombia, que invierten más en investigación y desarrollo tecnológico para integrar a sus sistemas productivos de café, conocimientos de genética y equipos de espectrofotometría de infrarrojo cercano (para detección de la composición química del grano) respectivamente, como parte de sus políticas agrarias de apoyo a la caficultura (Nahuamel, 2018; FNC, 2018). En este contexto, el objetivo del trabajo es definir un paquete tecnológico usado en el procesamiento de café verde peruano con características de café especial para exportación, bajo el enfoque de la gestión tecnológica, que podría utilizarse para un diagnóstico de la gestión tecnológica existente, de las necesidades específicas del caso y de una prospectiva tecnológica que permita calzar las tecnologías con los requerimientos de la demanda (Roca, 2014; Solleiro y Castañón, 2016).

\section{MATERIALES Y MÉTODOS}

\section{Enfoque teórico}

El desarrollo de este trabajo partió de los conceptos de la gestión tecnológica propuestos por Solleiro y Castañón (2016), quienes la definen como el conjunto de herramientas y técnicas que permiten a una organización aprovechar adecuadamente los recursos con los que cuenta (personas, dinero, máquinas, información, entre otros) mediante la elaboración y ejecución de planes de innovación. Para el estudio de la tecnología, se toma al paquete tecnológico como unidad de análisis (ZarazúaSolleiro et al., 2009; Kuramoto, 2010; Raya-Parra et al., 2015; Napaico, 2015; Takashi-Muraoka et al., 2019), y para la elaboración de un producto, puede estar compuesto por cuatro tecnologías (Tabla 1).
Tabla1. Componentes del paquete tecnológico para la elaboración de un producto*.

\begin{tabular}{|c|c|}
\hline Componente & Definición \\
\hline $\begin{array}{l}\text { Tecnología } \\
\text { de producto }\end{array}$ & $\begin{array}{l}\text { Es la que implica cambios } \\
\text { significativos en las características de } \\
\text { los bienes con la idea de } \\
\text { comercializarlo en un nicho de } \\
\text { mercado determinado. Ejm.: normas, } \\
\text { especificaciones, requisitos generales } \\
\text { aplicables al producto. }\end{array}$ \\
\hline $\begin{array}{l}\text { Tecnología } \\
\text { de equipo }\end{array}$ & $\begin{array}{l}\text { Se refiere a los bienes de capital } \\
\text { necesarios para producir un bien } \\
\text { Contiene al "hardware". Ejm: equipos, } \\
\text { implementos. }\end{array}$ \\
\hline $\begin{array}{c}\text { 3Tecnología } \\
\text { de proceso }\end{array}$ & $\begin{array}{l}\text { Es la que involucra cambios } \\
\text { significativos para combinar insumos } \\
\text { y bienes de capital de manera } \\
\text { adecuada para producir un bien. Se } \\
\text { relaciona a: condiciones, } \\
\text { procedimientos y/o formas para } \\
\text { combinar estos insumos y bienes de } \\
\text { capital. }\end{array}$ \\
\hline $\begin{array}{l}\text { Tecnología } \\
\text { de operación }\end{array}$ & $\begin{array}{l}\text { Se refiere al know-how o el } \\
\text { "software" necesario para asegurar la } \\
\text { calidad, confiabilidad, seguridad } \\
\text { física y durabilidad de la unidad de } \\
\text { producción y el producto. Se relaciona } \\
\text { a las normas y procedimientos } \\
\text { aplicables a las tecnologías de: } \\
\text { producto, equipo y proceso. }\end{array}$ \\
\hline
\end{tabular}

* Elaboración propia basada en las definiciones de Waissbluth et al. (1990) y Edquist (1997) citadas en Zarazúa-Solleiro et al. (2009).

\section{Metodología}

El estudio realizado fue de carácter exploratoriodescriptivo, auxiliado por entrevistas semiestructuradas a cinco profesionales, conocedores del procesamiento de productos agrícolas y de la perspectiva de la gestión tecnológica, como herramienta de análisis y metodología de intervención.

\section{Área de estudio}

El estudio se realizó sobre el procesamiento de café verde para exportación con características de café especial. El procesamiento se caracteriza como una etapa clave en el sistema productivo para obtener una buena calidad del café, pues ésta no está dada por un sólo factor (ubicación geográfica, variedad, prácticas agrícolas, etc.), sino por el cuidado que se tenga en todo el proceso (INIFAP, 2013). Esta calidad, lleva implícita su aplicabilidad para el 
comercio internacional (IICA, 2016). La calidad especial (Tabla 2) o gourmet se considera como una de las "características especiales" en el certificado de origen que debe emitirse para la exportación de café (ICO, 2018) hace que el café se distinga del común de los cafés y que los clientes estén dispuestos a pagar un precio superior (Farfán, 2007; Hernández-Gómez et al., 2018). Más aún, el presente trabajo se desarrolló sólo sobre el área de procesamiento, debido a que la consulta literaria sobre el uso de paquetes tecnológicos en el sector cafetalero y las entrevistas semi-estructuradas a los profesionales relacionados, revelaron que si bien se lleva a cabo una gestión tecnológica en la zona de producción (finca o fundo ) con un paquete integrado por tecnologías agrarias (tecnologías de producto y equipo ) y capacitaciones sobre el manejo agrícola (tecnologías de procesamiento y operación) propuesto por ingenieros agrónomos, en la zona de procesamiento (planta, sede, o sala), hasta dónde se conoce, en el país aún no se ha conformado un paquete tecnológico con la finalidad de gestionar las tecnologías de procesamiento de café verde con características de café especial para exportación. De estas, se identificaron las cualidades: físicas, sensoriales y culturales, obtenidas durante la ejecución de las operaciones del procesamiento, pues son éstas las que se pre-especifican para el procesamiento de café verde para exportación, y las que, según las tendencias del mercado, se recompensan mejor económicamente.

\section{Conformación y formulación del paquete tecnológico}

El proceso se realizó en tres partes. Primero se hizo una revisión literaria en bases de datos nacionales e internacionales (Instituto Nacional de Innovación Agraria; Universidad Nacional Agraria la Molina; Centro Nacional de Investigaciones de Café ; Información Técnica Económica Agraria; Google Académico) de artículos, libros, informes y tesis en temas relacionados al procesamiento de café verde en América Latina con fines de exportación, también de las especificaciones para obtener características de café especial en el producto final como resultado del procesamiento. Luego, con el material seleccionado, se identificaron las tecnologías genéricas (de producto, de equipo, de proceso y de operación), y se caracterizaron en una primera hoja de cálculo de Microsoft Excel 2016 como lo hicieron Zarazúa-Solleiro et al. (2009) y recomienda Napaico (2015).

Tabla 2. Calidad de los cafés especiales definida por sus características (Elaborado en base a Farfán (2007), Rhinehart (2009), Puerta (2011) y Dávila (2018)).

\begin{tabular}{|c|c|c|}
\hline $\begin{array}{l}\text { Características } \\
\text { físicas }\end{array}$ & $\begin{array}{l}\text { Forma, } \\
\text { tamaño, } \\
\text { humedad, } \\
\text { apariencia, } \\
\text { defectos }\end{array}$ & $\begin{array}{l}\text { Se controlan } \\
\text { durante el } \\
\text { procesamiento }\end{array}$ \\
\hline $\begin{array}{l}\text { Características } \\
\text { sensoriales }\end{array}$ & $\begin{array}{l}\text { Olfativas, } \\
\text { visuales y } \\
\text { gustativas }\end{array}$ & $\begin{array}{l}\text { Se preservan } \\
\text { durante el } \\
\text { procesamiento }\end{array}$ \\
\hline $\begin{array}{l}\text { Prácticas } \\
\text { culturales }\end{array}$ & $\begin{array}{l}\text { Recolección, } \\
\text { lavado, } \\
\text { secado }\end{array}$ & $\begin{array}{l}\text { Se controlan } \\
\text { durante el } \\
\text { procesamiento }\end{array}$ \\
\hline $\begin{array}{l}\text { Procesos } \\
\text { finales }\end{array}$ & $\begin{array}{l}\text { Tostón, } \\
\text { molienda, } \\
\text { preparación }\end{array}$ & $\begin{array}{l}\text { El tostador o } \\
\text { comprador expresa } \\
\text { las características } \\
\text { remanentes del } \\
\text { procesamiento. }\end{array}$ \\
\hline
\end{tabular}

Finalmente, para sustentar la definición de cada una de ellas y su importancia en conjunto, como paquete tecnológico para fines de gestión; en una segunda hoja de cálculo, se capturaron: el conocimiento, experiencia y percepción de los profesionales interrogados sobre este tema con un protocolo de entrevista semiestructurada (Silbey, 2020). Luego, se organizó la información en una matriz (Tabla 3), considerando en las columnas: nombre y apellidos completos, lugar y área de trabajo localidad, años de experiencia, definición y uso de cada tecnología propuesta en el paquete, posibles usos que se le puede dar en su conjunto, para beneficio de la agroindustria exportadora peruana y se procedió a su análisis bajo el enfoque de la gestión tecnológica para incorporar a la conformación final del paquete. 
Pajuelo, I. \& Huerta, L. (2021). Anales Científicos. 82(1), 171-179. DOI. 10.21704/ac.v82i1.1752

Tabla 3. Entrevistas.

\begin{tabular}{|c|c|c|c|c|}
\hline Fecha & $\begin{array}{l}\text { Nombre y } \\
\text { Apellidos }\end{array}$ & Lugar de Trabajo & Área de trabajo & Aporte \\
\hline 04-07-20 & $\begin{array}{l}\text { Carmen Silva } \\
\text { Cubillas }\end{array}$ & $\begin{array}{l}\text { Universidad Le Cordon } \\
\text { Bleu-Lima }\end{array}$ & $\begin{array}{l}\text { Facultad de } \\
\text { Ciencia de los } \\
\text { Alimentos }\end{array}$ & $\begin{array}{l}\text { Descripción de las tecnologías usadas en el } \\
\text { procesamiento de una materia prima hasta } \\
\text { un producto final en la agroindustria. }\end{array}$ \\
\hline $05-07-20$ & $\begin{array}{l}\text { Miki } \\
\text { Choquehuanca } \\
\text { Cruz }\end{array}$ & $\begin{array}{l}\text { Empresa Transnacional } \\
\text { Ed\&Man Volcafe Perú } \\
\text { S.A.-Jaén }\end{array}$ & $\begin{array}{l}\text { Área de } \\
\text { Sostenibilidad } \\
\text { Comercial, } \\
\text { Técnica y } \\
\text { Certificación }\end{array}$ & $\begin{array}{l}\text { Descripción de las tecnologías usadas } \\
\text { durante el procesamiento del café verde. }\end{array}$ \\
\hline 09-07-20 & $\begin{array}{l}\text { Luis Hernán } \\
\text { Nova Moscoso }\end{array}$ & $\begin{array}{l}\text { Fundo Café Diablo Fuerte } \\
\text { S.A. Oxapampa }\end{array}$ & Emprendimiento & $\begin{array}{l}\text { Descripción de las tecnologías usadas } \\
\text { durante el procesamiento de café tostado } \\
\text { molido. }\end{array}$ \\
\hline $10-07-20$ & $\begin{array}{l}\text { Grover } \\
\text { Augusto } \\
\text { Chuquipiondo } \\
\text { Pérez }\end{array}$ & $\begin{array}{l}\text { Centro de Innovación } \\
\text { Productiva y } \\
\text { Transferencia } \\
\text { Tecnológica (CITE)- } \\
\text { Selva Central }\end{array}$ & Dirección & $\begin{array}{l}\text { Descripción de las tecnologías usadas en la } \\
\text { planta de procesamiento agroindustrial. }\end{array}$ \\
\hline $11-07-20$ & $\begin{array}{l}\text { Monique } \\
\text { Muñoz Mosto }\end{array}$ & $\begin{array}{l}\text { Instituto de Desarrollo } \\
\text { Agroindustrial de la } \\
\text { Universidad Agraria La } \\
\text { Molina (INDDA)-Lima }\end{array}$ & $\begin{array}{l}\text { Dirección } \\
\text { Ejecutiva }\end{array}$ & $\begin{array}{l}\text { Descripción de la actual gestión } \\
\text { tecnológica en la agroindustria peruana. }\end{array}$ \\
\hline
\end{tabular}

\section{RESULTADOS Y DISCUSIÓN}

El paquete tecnológico conformado para el procesamiento de café verde agrupa un total de setenta y nueve tecnologías categorizadas por tipo de tecnología, distribuidas de la siguiente forma: (i) tecnología de producto, trece tecnologías, (ii) tecnología de equipo, trece tecnologías, (iii) tecnología de proceso, diez tecnologías y (iv) tecnología de operación, cuarenta y tres tecnologías (Tabla 4). La distribución absoluta de las tecnologías por categoría no es homogénea; la tecnología de producto se ve limitada por desconocimiento de otras especificaciones como el material de empaque al final del procesamiento. Además, la mayoría de especificaciones se dan en la zona de cultivo por los requerimientos de la certificación orgánica, que es la más usada y conocida en el Perú; las tecnología de equipo y proceso se ven limitadas por el presupuesto e inaccesibilidad a la información por la escasez de difusión tecnológica; la tecnología de operación sobrepasa a los demás, porque representa el conocimiento de las buenas prácticas que se obtiene en la capacitación; el servicio más ofrecido como parte de programas de transferencia tecnológica en el país.
Cabe señalar que, pese a la descripción (Tabla 1) y cuantificación de cada tipo de tecnología, la mayoría de estas tecnologías son genéricas, pues en el trabajo de campo, cada una de ellas puede registrar tantas variaciones como zonas de procesamiento de café verde se encuentren. Pueden variar según las especificaciones del sistema de beneficio, de los volúmenes de producción y del contrato de exportación. Lo anterior, se infirió inicialmente de la revisión literaria y luego se confirmó en las cinco entrevistas realizadas.

Kuramoto (2010) señala que el éxito exportador peruano, radica en la adopción de un paquete tecnológico que les permita producir de acuerdo con los requerimientos del mercado. En este trabajo se identificaron las operaciones de procesamiento de café verde con las que se obtienen las características de cafés especiales (Tabla 5) definidas por Farfán (2007) y se determinó que, dentro del paquete conformado, intervienen un total de 76 tecnologías (Tabla 6). De esta manera, una mejora en el paquete para generar valor agregado en el producto puede abordarse al conocer las tecnologías que producen las características del producto, y luego adoptar el 
tipo de tecnología que se adapte mejor a sus recursos, capacidades y objetivos teniendo en cuenta la tecnología que genere el máximo rendimiento del paquete al usarse con las demás (Raya-Parra et al., 2015).

Tabla 4. Paquete tecnológico para el procesamiento de café verde peruano (Dávila, 2018; Benavente, 2018; Gómez, 2018).

\begin{tabular}{|c|c|c|c|c|c|}
\hline $\begin{array}{c}\text { Operaciones del } \\
\text { Procesamiento } \\
\text { Vía Húmeda }\end{array}$ & $\begin{array}{c}\text { Tecnología } \\
\text { de producto } \\
\text { Especificaciones }\end{array}$ & $\begin{array}{l}\text { Tecnología } \\
\text { de equipo } \\
\text { "hardware" }\end{array}$ & $\begin{array}{c}\text { Tecnología de } \\
\text { proceso } \\
\text { Combinaciones }\end{array}$ & $\begin{array}{l}\text { Tecnología de } \\
\text { operación } \\
\text { "Know how" }\end{array}$ & $\begin{array}{l}\mathrm{N}^{\circ} \text { total de } \\
\text { tecnologías }\end{array}$ \\
\hline 1. Selección & 3 & 2 & 3 & 3 & 11 \\
\hline 2. Despulpado & 1 & 1 & 1 & 7 & 10 \\
\hline 3. Fermentación & 2 & 2 & 1 & 5 & 10 \\
\hline 4. Lavado & 0 & 2 & 1 & 5 & 8 \\
\hline 5.Clasificación & 1 & 1 & 0 & 1 & 3 \\
\hline 6. Secado & 1 & 1 & 1 & 7 & 10 \\
\hline 7. Control de calidad & 1 & 0 & 1 & 3 & 5 \\
\hline 8. Almacenamiento I & 1 & 0 & 0 & 2 & 3 \\
\hline 9. Trillado & 0 & 1 & 1 & 1 & 3 \\
\hline $\begin{array}{l}\text { 10. Empacado y } \\
\text { Almacenamiento II }\end{array}$ & 3 & 3 & 1 & 9 & 16 \\
\hline $\mathrm{N}^{\circ}$ Total de tecnologías & 13 & 13 & 10 & 43 & 79 \\
\hline
\end{tabular}

Tabla 5. Características de cafés especiales obtenidas durante el procesamiento (Modificado de Farfán (2007), Rhinehart (2009), Puerta (2011), Dávila (2018), Benavente (2018) y Gómez (2019)).

\begin{tabular}{|c|c|c|}
\hline \multicolumn{2}{|c|}{$\begin{array}{l}\text { Características de cafés } \\
\text { especiales }\end{array}$} & \multirow{2}{*}{$\begin{array}{l}\text { Operaciones de } \\
\text { procesamiento } \\
\text { Selección, despulpado, } \\
\text { fermentación, } \\
\text { clasificación, secado, } \\
\text { almacenamiento I, } \\
\text { empacado y } \\
\text { almacenamiento II }\end{array}$} \\
\hline Físicas & $\begin{array}{l}\text { Forma, } \\
\text { tamaño, } \\
\text { humedad, } \\
\text { apariencia, } \\
\text { defectos }\end{array}$ & \\
\hline Sensoriales & $\begin{array}{l}\text { Olfativas, } \\
\text { visuales, } \\
\text { gustativas }\end{array}$ & $\begin{array}{l}\text { Control de calidad, } \\
\text { almacenamiento I }\end{array}$ \\
\hline Culturales & $\begin{array}{l}\text { Recolección, } \\
\text { lavado, } \\
\text { secado }\end{array}$ & Lavado, secado \\
\hline
\end{tabular}

Por otra parte, Zarazúa- Solleiro et al. (2008) conformaron un paquete tecnológico para estudiar la dinámica de la innovación en las empresas frutícolas del sistema-producto guayaba en México, bajo el enfoque del aprendizaje tecnológico. De manera que, definió una tecnología material o tangible (tecnologías de producto y equipo) y una tecnología de conocimiento o intangible (tecnología de proceso, operación y organizacional) como sus componentes. Al conformar y estructurar el paquete aludió a la importancia a la vigilancia del entorno en el proceso de innovación y transferencia de tecnología, lo cual indica que la conformación del paquete en este estudio es válida y puede expandirse con el enfoque sugerido para caracterizar los esquemas de innovación tecnológica y su transferencia en la agroindustria peruana exportadora de café verde o de otras líneas de productos.

Tablas 6. Tecnologías usadas en el procesamiento de café verde para la obtención de características de cafés especiales para exportación (Modificado de Farfán (2007), Rhinehart (2009), Puerta (2011), Dávila (2018), Benavente (2018) y Gómez (2019)).

\begin{tabular}{|c|c|c|c|}
\hline Operaciones & $\begin{array}{l}\mathrm{N}^{\circ} \\
\text { Tecnologías }\end{array}$ & $\begin{array}{l}\text { Tecnologías por } \\
\text { categoría }\end{array}$ & \\
\hline Selección & 11 & & \\
\hline Despulpado & 10 & & \\
\hline Fermentación & 10 & & \\
\hline Lavado & 8 & & \\
\hline Clasificación & 3 & & \\
\hline Secado & 10 & T. de producto & 12 \\
\hline Control de calidad & 5 & T. de equipo & 12 \\
\hline Almacenamiento I & 3 & T. de proceso & 9 \\
\hline Trillado & 0 & T. de operación & 42 \\
\hline $\begin{array}{l}\text { Empacado y } \\
\text { Almacenamiento II }\end{array}$ & 16 & $\begin{array}{l}\mathrm{N}^{\circ} \text { total de } \\
\text { tecnologías }\end{array}$ & 76 \\
\hline $\begin{array}{l}\mathrm{N}^{\circ} \text { total de } \\
\text { tecnologías }\end{array}$ & 76 & & \\
\hline
\end{tabular}


En Perú, Napaico (2015) indica que los alcances tecnológicos aplicables a la agroindustria cafetalera de programas e instituciones son limitados en presupuesto y personal calificado. Por ello, sugiere que con un estudio eficiente en el que se clasifique la tecnología de acuerdo a su composición para poder identificar las tecnologías necesarias para la elaboración del producto, podría hacerse una mejor gestión de los recursos que ya se tiene. El enfoque de la gestión tecnológica bajo el que se conformó el paquete para el procesamiento de café verde puede tener dicha utilidad, teniendo en cuenta que los resultados de su uso siempre dependerán del contexto (Mwangi y Kariuki, 2015).

Es importante señalar que, en los países en vías de desarrollo, se recomienda el uso de paquetes tecnológicos para el estudio de la adopción de tecnologías, pero aún muchos casos de estudio se enfocan únicamente en el estudio de una sola tecnología (Takashi-Muraoka et al., 2019). La conformación de un paquete para el procesamiento de café verde peruano se ajusta a esta recomendación con las limitaciones de especificidad en las tecnologías identificadas, por ser un estudio exploratorio- descriptivo.

\section{CONCLUSIÓN}

Se caracterizó un paquete tecnológico (con tecnologías genéricas) para el procesamiento de café verde peruano con características de cafés especiales para exportación de manera exploratoria-descriptiva bajo el enfoque de la gestión tecnológica. Por lo tanto, la conformación del paquete en este estudio carece de especificidad técnica como: parámetros para las tecnologías de producto y proceso; características concretas para la tecnología de equipo y ajuste del paquete para el volumen de producción de la zona de procesamiento. Sin embargo, la revisión literaria y las entrevistas revelaron su potencial utilidad para: generar valor agregado para el usuario al integrar definiciones y objetivos claros; comparar el rendimiento del uso conjunto de las tecnologías entre las diferentes zonas de procesamiento que tiene una sola empresa mediante indicadores; identificar concretamente la tecnología que permitiría generar durante el procesamiento del producto una característica específica que se demanda en el mercado internacional; conformar paquetes tecnológicos para cada una de las líneas de producto en la agroindustria para monitoreo y auditorías; evaluación de costos entre tecnologías o paquetes para toma de decisiones y combinación estratégica de las tecnologías existentes en la zona de procesamiento.

\section{Conflictos de intereses}

Los autores firmantes del presente trabajo de investigación declaran no tener ningún potencial conflicto de interés personal o económico con otras personas $\mathrm{u}$ organizaciones que puedan influir indebidamente con el presente manuscrito.

\section{Contribuciones de los autores}

Preparación y ejecución: IPT, LHC; Desarrollo de la metodología: IPT, LHC; Concepción y diseño: IPT, LHC; Edición del artículo: IPT, LHC; Supervisión del estudio: LHC.

\section{LITERATURA CITADA}

- Aire, J. (2019). Factores de la oferta exportable en las empresas agroexportadoras de café en la provincia de Chanchamayo, 2018. Tesis de pregrado, Universidad Continental, Huancayo. Perú. 245 p. Disponible en: https://repositorio.continental.edu.pe/handle/20. $500.12394 / 6045$

- Benavente, K. (2018). Propuesta de un proceso de calidad en la producción de café en Oxapampa-Villa Rica basado en la gestión por procesos para aumentar la productividad. Tesis Ingeniería Industrial, Universidad Peruana de Ciencias Aplicadas, Lima, Perú. 330 p. Disponible en: https://repositorioacademico.upc.edu.pe/handle/ $10757 / 624415$ ?show=full\&locale-attribute $=e s$

- Bitzer, V., Glasbergen, P., \& Arts, B. (2013). Exploring the potential of intersectoral partnerships to improve the position of farmers in global agri-food chains: findings from the coffee sector in Peru. Agric. Human Values, 30 (1),5-20. https://doi.org/10.1007/s10460-0129372-z

- Dávila, J. (2018). Influencia de parámetros de tostado de café especial sobre el puntaje de taza 
obtenido mediante un panel de jueces certificados. Tesis Magister Scientiae en Tecnología de Alimentos, Universidad Nacional Agraria La Molina, Lima. Perú. 212 p. Disponible en: http://repositorio.lamolina.edu.pe/bitstream/han dle/UNALM/3401/davila-romero-juancarlos.pdf?sequence $=1$ \&isAllowed $=\mathrm{y}$

- Delgado, W. (2009). Caracterización del proceso de transferencia y adopción tecnológica de pequeños y medianos productores de cebolla (Allium cepa L.) en el municipio de Pasca (Cundinamarca). Tesis de Magíster en Desarrollo Rural, Pontificia Universidad Javeriana, Bogotá. Colombia. 119 p. Recuperado de:

https://repository.javeriana.edu.co/bitstream/han dle/10554/134/eam1.pdf?sequence=2\&isAllowe $\mathrm{d}=\mathrm{y}$

- Farfán, F. (2007). Cafés especiales. En: CENICAFÉ. Sistemas de Producción de Café en Colombia. Digital repository of the National Coffee Research Centre. Colombia. 234-254 p. Recuperado de: http://hdl.handle.net/10778/720

- FNC [Federación Nacional del Café de Colombia]. (2018). Informe de Gestión. Recuperado de: https://federaciondecafeteros.org/app/uploads/2 019/10/Informe_Gestion_2018_compressed1.pdf

- Gómez, J. (2019). Modelación del procesamiento agroindustrial del café desde la fruta hasta el café oro. Informe de Proyecto Final de Graduación, Universidad de Costa Rica, Ciudad Universitaria Rodrigo Facio. Costa Rica.100 p. Disponible en: http://www.kerwa.ucr.ac.cr/handle/10669/7979 4

- Grabs, J., \& Ponte, S. (2019). The evolution of power in the global coffee value chain and production network. Journal of Economic Geography 19(1), 803-828. doi:10.1093/jeg/lbz008

- Hernández-Aguilera, J., Gómez, M., Rodewald, A., Rueda, X., Anunu, C., Bennett, R., \& van Es, H. (2018). Quality as a Driver of Sustainable Agricultural Value Chains: The Case of the Relationship Coffee Model. Bus. Strateg.
Environ $\quad 27 \quad$ (2), $179-198$. https://doi.org/10.1002/bse.2009

- IICA. [Instituto Interamericano de Cooperación para la Agricultura]. (2016). La situación y tendencias de la producción de café en América Latina y el Caribe. Disponible en: https://camcafeperu.com.pe/admin/recursos/pub licaciones/La-situacion-tendencias-produccioncafe-America-Latina-y-Caribe.pdf

- INIA. [Instituto Nacional de Innovación Agraria]. (2013). Centro Piloto de Investigación Adaptativa y Capacitación en Café. Disponible en: Http://repositorio.inia.gob.pe/handle/inia/974

- INIA. [Instituto Nacional de Innovación Agraria]. (2016). Guía Metodológica para la Transferencia de Tecnología Agraria. Disponible en: Http://repositorio.inia.gob.pe/handle/inia/479

- INIFAP. [Instituto Nacional de Investigaciones Forestales, Agrícolas y pecuarias]. (2013). Paquete Tecnológico para el Cultivo de Café Sierra Huasteca Potosina. Disponible en: http://www.inifapcirne.gob.mx/Biblioteca/Paqu etes2012/81.pdf

- JNC. [Junta Nacional del Café]. (2019). El Cafetalero. Disponible en: https://juntadelcafe.org.pe/revista-cafetalero/

- Kuramoto, J. (2010). Prácticas exitosas de innovación empresarial y comportamiento tecnológico sectorial. En: Peña, L. Innovación empresarial y comportamiento tecnológico sectorial: experiencias exitosas y estudios de casos. Lima: CIES; FINCYT.5-19 p. Disponible en:

https://innovateperu.gob.pe/fincyt/doc/INFOR MES_CIES/Estudios\%20Sintesis.pdf

- MINAGRI. [Ministerio de Agricultura e Irrigación]. (2018). Plan Nacional de Acción del Café Peruano 2018-2030. Disponible en: https://www.undp.org/content/dam/peru/docs/P ublicaciones \%20medio\%20ambiente/PNACafe $\% 20$ (pliegos)\%20180ct2018\%20(1).pdf

- Mwangi, M., \& Kariuki, S. (2015). Factors Determining of New Agricultural Technology by Smallholder farmers in Developing Countries. Journal of Economics and Sustainable Development 6(5), 208-216. Disponible en: 
https://www.iiste.org/Journals/index.php/JEDS/ article/view/20710/21632

- Nahuamel, E. (2018). Factores competitivos para el desarrollo de la caficultura peruana. Pensamiento Crítico 23 (2), 97114.doi.org/10.15381/pc. v23i2.15806

- Napaico, B. (2015). La influencia de la innovación tecnológica en la producción y procesamiento del café en la provinciaChanchamayo. Tesis de Pregrado, Universidad Nacional del Centro del Perú, Huancayo, Perú. 228p. Disponible en: http://repositorio.uncp.edu.pe/handle/UNCP/135

- OECD. [Organization for Economic Cooperation and Development]. (2011). OECD Reviews of Innovation Policy: Peru. Disponible en: https://www.oecd-ilibrary.org/science-andtechnology/oecd-reviews-of-innovation-policyperu-2011_9789264128392-en

- OIC. [Organización Internacional del Café]. (2018). Reglamento de Estadística Certificados de Origen. Londres 13 de abril de 2018, pp: 1-8. Disponible en: http://www.ico.org/documents/cy2017-18/icc102-9-r1c-rules-certificates-origin-final.pdf

- Palacios, L. (2014). Proyecto de cooperaciónResponsabilidad social corporativa: caracterización del café de Satipo. Trabajo de Fin de Grado, Universidad de La Rioja, Logroño. España. 57 p. Disponible en: https://biblioteca.unirioja.es/tfe_e/TFE000859.pdf

- PNUD. [Programa de las Naciones Unidas para el Desarrollo]. (2017). Línea Base del Sector Café en el Perú. Disponible en: https://www.pe.undp.org/content/peru/es/home/ library/environment_energy/linea-de-base-delsector-cafe-en-el-peru.html

- Puerta, G. (2011). Composición química de una taza de café. Avances Técnicos Cenicafé. Centro Nacional de Investigaciones de Café. Manizales, Colombia. Disponible en: https://www.cenicafe.org/es/publications/avt04142.p df

- Raya, V.; Parra, M. \& Cid, M. (2015). Evaluación de un nuevo paquete tecnológico para producción de tomate de exportación. Revista Agropecuaria 1 (22), 77-82. Disponible en:

http://anuariosatlanticos.casadecolon.com/index .php/GRANJA/article/view/9942

- Rhinehart, R. (2009). What is a specialty coffee? SCAA Articles. California, USA. Available in: https://scanews.coffee/2017/03/17/what-isspecialty-coffee/

- Roca, T. (2014). Políticas y factores que contribuyen a la transferencia de tecnología en organizaciones del Perú. Revista Venezolana de Gerencia, 19 (68), 639-669. Disponible en: https://www.redalyc.org/articulo.oa?id=290328 19003

- Silbey, S. (2020). Qualitative Research Methods: Conversational Interviewing. [Virtual Lessons]. Massachusetts Institute of Technology, Perú, Lima. Available in: https://www.edx.org/course/qualitativeresearch-methods-conversational-interv

- Solleiro, J., \& Castañón, R. (2016). Gestión tecnológica: conceptos y prácticas. 2da Edición. Plaza y Valdés Editores, México. 410 p. Disponible en: http://cambiotec.org.mx/site/wpcontent/uploads/2017/09/E-Libro-

Gestio\%CC\%81n-2.pdf

- SUNAT. [Superintendencia Nacional de Aduanas y Administración Tributaria]. (2020). Estadísticas y Estudios (marzo 2020). Información aduanera exportaciones. [Exportaciones definitivas de principales subpartidas nacionales por sector económico]. Disponible en: http://www.sunat.gob.pe/estadisticasestudios/ex portaciones.html

- Takashi, K., Muraoka, R., \& Otsuka, K. (2019). Technology adoption, impact, and extension in developing countries' agriculture: A review of the recent literature. Agricultural Economics 51 (1): 31-45. doi.org/10.1111/agec.12539

- Zarazúa, J., Solleiro, J., Altamirano, R., Castañón, R., \& Rendón, R. (2009). Esquemas de innovación tecnológica y su transferencia en el agro empresas frutícolas del estado de Michoacán. Estudios Sociales. Revista de Alimentación Contemporánea y Desarrollo Regional, 17(34), 38-71. Disponible en: https://www.redalyc.org/articulo.oa?id=4171150 